\title{
The assembly of a composite based on nano-sheet graphene oxide and montmorillonite
}

\author{
Yu-Xiu An ${ }^{1} \cdot$ Wei-Jia Qu' $\cdot$ Pei-Zhi Yu $\cdot$ Jian-Guo Lü ${ }^{1}$
}

Received: 27 June 2017 / Published online: 8 February 2018

(c) The Author(s) 2018. This article is an open access publication

\begin{abstract}
Graphene oxide (GO) nano-sheets were synthesized using a modified Hummers' method from graphite powder. The Raman spectrum of GO displayed a D-band at $1359 \mathrm{~cm}^{-1}$ and a G-band at $1594 \mathrm{~cm}^{-1}$. The $I_{D} / I_{G}$ value of GO was calculated to be 0.97 , suggesting the formation of new $s p^{2}$ clusters upon reduction. A method was designed to investigate the assembly of the GO/montmorillonite (MMT) composite. After the addition of GO, the typical peaks of montmorillonite in FT-IR spectra shifted, indicating the assembly between GO and MMT. The D-band and G-band reduced sharply in the GO/MMT composite. More importantly, the D-band $\left(1344 \mathrm{~cm}^{-1}\right)$ and G-band $\left(1574 \mathrm{~cm}^{-1}\right)$ shifted significantly and the $I_{\mathrm{D}} /$ $I_{\mathrm{G}}$ value of the GO/MMT composite was calculated to be 1.13, showing a change in the GO structure. In the addition of $0.04 \mathrm{wt} \%$ GO to MMT, the value of interlayer space $(d)$ was up to $13.0 \AA$ measured by XRD due to the insertion of GO into MMT. The evident increases in contents of carbon atoms (26.59\%) and nitrogen atoms (3.44\%) indicate that GO was successfully combined with MMT. The nano-pores and clay sheets were not observed in the SEM image of GO/clay, but obvious wrinkles, while flexible sheets were observed in the typical scanning electron microscopy images of GO. This further proves that GO was combining with clay. The TEM image shows that the GO nano-sheets were tiled on the surface of MMT sheets. This observation suggests that a stable assembly structure was formed between GO sheets and MMT sheets. The change in particle size of MMT with the addition of GO shows that interaction occurred between GO sheets and MMT sheets, which was further confirmed by the results of zeta potential. Adsorption and insertion were the main mechanisms to assemble GO and MMT.
\end{abstract}

Keywords Drilling fluid · Graphene oxide $\cdot$ Montmorillonite $\cdot$ Wellbore stability

\section{Introduction}

With economic development and increasing demand for energy, as well as the depletion of conventional oil and gas reserves, more and more attention has been paid to unconventional oil and gas resources (Rajnauth 2012). Shale gas is considered to be an unconventional source due to shale's typically low porosity and low permeability. Shale gas has been produced at industrial scale in the USA, and the shale gas revolution has swiftly erupted around the world. It has been estimated that the drilled formations are

Edited by Yan-Hua Sun

Jian-Guo Lü

ljg@cugb.edu.cn

1 School of Engineering and Technology, China University of Geosciences (Beijing), Beijing 100083, China more than $75 \%$ shale (Josh et al. 2012). It is very important to maintain wellbore stability during shale formation drilling (Al-Bazali 2011; Bybee 2009; Díaz-Pérez et al. 2007; $\mathrm{Hu}$ et al. 2016; Riley et al. 2012). Ninety percentage of wellbore stability problems are caused by shale formations. Shale formations consist of consolidated clay minerals and contain native fractures and nano-pores. This results in their ultra-low permeability (van Oort 2003; Zhang et al. 2013; Zhong et al. 2011) and serious clay swelling when encountering water, a main factor causing hole collapse, wellbore weakening, and stuck pipes in drilling (Zhong et al. 2015). The composition of clay minerals is a major factor contributing to swelling capability as some clay minerals are non-swelling. For example, smectite is a typical swelling-type clay mineral, but illite and chlorite are non-swelling minerals. The swelling of smectite results in an increase in swelling pressure and is followed by a series of wellbore instability problems. Long horizontal 
segments aggravate wellbore instability during drilling shale formations due to the swelling of clay minerals. Montmorillonite is a member of the smectite group, and smectite and montmorillonite are often not distinguished. Controlling the swelling of montmorillonite is an effective solution to instability of shale formations.

Oil-based drilling fluids are effective in drilling shale formations because the clay minerals do not hydrate in this type of drilling fluids (Bunger et al. 2014). However, the oil-based drilling fluid is expensive and not environmentally friendly (Shivhare and Kuru 2014; Simpson et al. 1995; Young and Friedheim 2013). Governments and environmental departments in developed countries have introduced stricter regulations to treat all kinds of drilling waste. Increasingly stricter environmental regulations will require more environmentally friendly water-based drilling fluids. Several issues need to be resolved before waterbased drilling fluids can be used. The diffusion of water into shale results in swelling, delaminating, and fracturing (van Oort 1994). Otherwise, horizontal drilling requires drilling fluids with better performance to achieve wellbore stability due to the long period of interaction of waterbased drilling fluids with shale formations (Mohiuddin et al. 2007). Inhibitors are commonly used in drilling fluids to prevent clay swelling. In recent years, Zhong et al. 2012 proposed that poly(oxypropylene)-amidoamine (POAA) was a potential shale inhibitor in water-based drilling fluids. Qu et al. (2009) investigated the performance of polyoxyalkyleneamine to prevent shale hydration and swelling, but the inhibiting ability was insufficient when polyoxyalkyleneamine was used in water-based fluids during drilling shale formations. Shadizadeh et al. (2015) reported a novel nonionic surfactant extracted from a plant leaf for inhibiting shale hydration. An et al. (2015) evaluated the inhibition of chitosan quaternary ammonium salt (HTCC), and its inhibiting ability was better than polyether amine, but the cost of HTCC was high. The commonly used inhibitors include $\mathrm{KCl}, \mathrm{CaCl}_{2}, \mathrm{NH}_{4} \mathrm{Cl}$, modified gilsonites, and asphalts (Gholizadeh-Dooechaly et al. 2009; Xiong et al. 2012). However, these approaches have some disadvantages, for example, short effectiveness, toxicity, low heat and salinity tolerance and $\mathrm{pH}$ sensitivity and expense. The inhibiting ability of inhibitors was enough in drilling traditional reservoirs, but the hydration of watersensitive shale was not completely controlled. A series of wellbore instability problems have happened during drilling shale formations with water-based drilling fluids. How to enhance the inhibiting ability of inhibitors is a crucial solution to wellbore instability of shale formations.

Graphene materials have been widely used in many fields because of their atom-thick two-dimensional conjugated structures, large specific surface area, and high conductivity (Geim 2009; Novoselov et al. 2005;
Stankovich et al. 2006; Stoller et al. 2008; Wu et al. 2010; $\mathrm{Xu}$ et al. 2008). Up to now, graphene materials were prepared by epitaxial growth (Pearce et al. 2011), chemical vapor deposition (Reina et al. 2009), mechanical exfoliation (Robinson et al. 2008), and thermal or chemical modification (Park and Ruoff 2009). Graphene oxide (GO) has been used for preparing graphene materials. Furthermore, the oxygenated groups provide GO sheets with reaction capacity and the possibility of chemical modifications (Dreyer et al. 2010). Several researchers investigated the interaction of graphene composites ( $\mathrm{Xu}$ et al. 2013; Zhang et al. 2011; Zuo et al. 2017). Kosynkin and co-workers synthesized graphene oxide as a new fluid loss agent (Kosynkin et al. 2012). This newly synthesized fluid loss agent performed well compared with polymer-based fluid loss agents. Tour et al. (2011) used graphene and modified graphene materials to stabilize shale formations during drilling operations, but they did not ascertain interaction mechanisms and did not provide experimental data to show the plugging capability of these graphene materials. An et al. (2016) reported the application of graphene and modified graphene material to inhibit the swelling of shale formations by plugging nano-pores in shales, but the detailed mechanism has been not studied. This paper is to further study the interaction mechanism between GO nano-sheet and montmorillonite.

It is novel to develop mature graphene materials for inhibiting the hydration of shales. This basic research into graphene oxide and montmorillonite is an important step to develop mature graphene materials.

\section{Experimental}

\subsection{Materials}

Montmorillonite (MMT) was purchased from Huawei Company. Graphite powder (325 mesh) was purchased from Alfa Reagent Company. Potassium permanganate $\left(\mathrm{KMnO}_{4}\right)$, sodium nitrate $\left(\mathrm{NaNO}_{3}\right)$, concentrated sulfuric acid $\left(\mathrm{H}_{2} \mathrm{SO}_{4}, 98 \%\right)$, hydrogen peroxide $\left(\mathrm{H}_{2} \mathrm{O}_{2}\right)$, and other reagents were commercially available. All the reagents were used without further purification.

\subsection{Methods}

\subsubsection{Synthesis of graphene oxide (GO)}

GO was synthesized using a modified Hummer's method from graphite powder, and the detailed process was reported in the literature (Hummers and Offeman 1958; Xu et al. 2009). Six grams of graphite powder was put into $120 \mathrm{~mL}$ of cold concentrated $\mathrm{H}_{2} \mathrm{SO}_{4}\left(0^{\circ} \mathrm{C}\right)$. Then, $\mathrm{NaNO}_{3}$ 
( $3 \mathrm{~g}$ ) was added to the concentrated $\mathrm{H}_{2} \mathrm{SO}_{4}$ while stirring. After the $\mathrm{NaNO}_{3}$ was completely dissolved, $15 \mathrm{~g} \mathrm{KMnO}_{4}$ was added slowly with vigorous stirring and the temperature of the mixture was kept below $20^{\circ} \mathrm{C}$ in an ice bath. The mixture was then stirred vigorously at $35{ }^{\circ} \mathrm{C}$ for $2 \mathrm{~h}$ and then gradually diluted with deionized water $(250 \mathrm{~mL})$. The addition of water was carried out in an ice bath to keep the temperature below $50{ }^{\circ} \mathrm{C}$. After adding all the $250 \mathrm{~mL}$ of deionized water, the mixture was stirred for $2 \mathrm{~h}$. The mixture was heated to $90{ }^{\circ} \mathrm{C}$ and kept for $15 \mathrm{~min}$. And then an additional $700 \mathrm{~mL}$ of deionized water was added to the solution and the mixture was cooled to room temperature. Shortly afterward $20 \mathrm{~mL}$ of $30 \% \mathrm{H}_{2} \mathrm{O}_{2}$ was added to the mixture, and the color of the bubbling mixture changed to brilliant yellow. The mixture was centrifuged at $8000 \mathrm{rpm}$ for $20 \mathrm{~min}$, and then, the precipitate was added to $1: 10 \mathrm{HCl}$ aqueous solution to remove metal ions. This was repeated three times followed by washing with deionized water to remove the acid. This operation was also repeated three times. Finally, the mixture was purified by dialysis for 1 week to remove the remaining metal species. The resulting homogeneous yellow-brown dispersion was obtained.

\subsubsection{Preparation of GO/MMT hybrids}

Firstly, $300 \mathrm{~mL} \mathrm{GO}$ dispersions of different concentrations (0.005 wt $\%, \quad 0.01 \mathrm{wt} \%, \quad 0.02 \mathrm{wt} \%, \quad 0.04 \mathrm{wt} \%$, and $0.08 \mathrm{wt} \%$ ) were prepared. Then, $9 \mathrm{~g}$ MMT was added to the GO dispersion to produce a GO/MMT dispersion, followed by vigorous stirring at $8000 \mathrm{rpm}$ for $30 \mathrm{~min}$. Then the dispersion was left to stand at $80{ }^{\circ} \mathrm{C}$ for $16 \mathrm{~h}$ to equilibrate the adsorption and hydration between MMT and GO. Thereafter, the dispersion was centrifuged at $10,000 \mathrm{rpm}$ for $15 \mathrm{~min}$. The precipitate was washed several times with deionized water until the upper liquid was clear. Finally, after being dried at $80{ }^{\circ} \mathrm{C}$ for $24 \mathrm{~h}$, the precipitate was ground into powder for XRD, XPS, FT-IR, and Raman spectra analysis.

\subsection{Structure characterization of GO/MMT hybrids}

\subsubsection{Fourier transform infrared spectroscopy (FT-IR) measurements}

FT-IR spectra of GO/MMT of different GO concentrations were recorded with a Magna-IR 560 spectrometer (Nicolet, USA) using the $\mathrm{KBr}$ disk method. The $\mathrm{KBr}$ disk (2 mg powdered sample and $200 \mathrm{mg} \mathrm{KBr}$ ) was prepared in a mold with a hydraulic press of $50 \mathrm{MPa}$.

\subsubsection{X-ray diffraction (XRD) measurements}

XRD spectra of the GO/MMT hybrid were recorded with a D8 advance diffractometer (Bruker, Germany). The scan range $(2 \theta)$ was from $0.5^{\circ}$ to $10^{\circ}$, and $\mathrm{Cu} \mathrm{K} \alpha_{1} \mathrm{X}$-radiation was used. Two grams of sample was ground to powder. This powder was poured into a mold and compacted by a glass sheet.

\subsubsection{X-ray photoelectron spectroscopy (XPS) measurements}

XPS analysis of GO/MMT was conducted on an ESCALAB 250 X-ray photoelectron spectrometer (Symer, UK). Two grams of sample was powdered. This sample was poured into a mold and compressed at $10 \mathrm{MPa}$ for $10 \mathrm{~s}$ by a hydraulic press. These compacted samples were dried at $80{ }^{\circ} \mathrm{C}$ for $24 \mathrm{~h}$. Spectral scans of C, N, O, Si, and Al were carried out.

\subsubsection{Scanning electron microscopy (SEM)}

A fresh shale sheet was put into a GO solution (0.001 wt $\%$ ) for $10 \mathrm{~h}$ at room temperature, and then the sheet was dried at $60{ }^{\circ} \mathrm{C}$ for $24 \mathrm{~h}$. These shale sheets were adhered to the special mold by adhesive tape, and then the mold was vacuum-coated with gold for $2 \mathrm{~min}$. Samples were imaged with a scanning electron microscope (Quanta 200F, FEI, Netherlands).

\subsubsection{Particle size analysis}

The particle size analysis of MMT/GO suspensions was performed by a Zetasizer Nano ZS instrument. A series of $0.1 \mathrm{wt} \%$ MMT suspensions were prepared and dispersed ultrasonically at room temperature for $20 \mathrm{~min}$. Different amounts of GO (0.001 wt \%, $0.005 \mathrm{wt} \%, 0.01 \mathrm{wt} \%$, and $0.02 \mathrm{wt} \%$ ) were added to the MMT aqueous suspensions and then shaken for $0.5 \mathrm{~min}$. A series of GO/MMT suspensions were obtained. These suspensions were allowed to stand at room temperature for $5 \mathrm{~h}$ to assemble enough.

\subsubsection{Zeta potential measurements}

The particle size analysis of GO/MMT suspensions was performed with a Zetasizer Nano ZS instrument. A series of $0.1 \mathrm{wt} \%$ MMT suspensions were prepared and dispersed ultrasonically at room temperature for $20 \mathrm{~min}$. Different amounts of GO (0.001 wt \%, $0.005 \mathrm{wt} \%, 0.01 \mathrm{wt} \%$, $0.02 \mathrm{wt} \%$ ) were added to the MMT aqueous suspensions and shaken by hand for $0.5 \mathrm{~min}$. A series of GO/MMT suspensions were obtained. These suspensions were allowed to stand at room temperature for $5 \mathrm{~min}$ to assemble enough. 


\subsubsection{Raman spectra analysis}

Raman spectra of GO and GO/MMT hybrid were recorded using a Renishaw Raman microscope (Evolution, HORIBA, France) with a 514-nm laser with $4.7 \mathrm{~mW}$ power.

\subsubsection{Transmission electron microscopy (TEM)}

Samples were examined using a F20 transmission electron microscope (JEOL, Japan). A MMT suspension (0.001 wt $\%)$ was prepared by dispersing MMT powder in water ultrasonically at room temperature for $20 \mathrm{~min}$. A $0.001 \mathrm{wt} \%$ GO dispersion was added to the MMT aqueous suspension to make a GO/MMT suspension. One drop of the GO/MMT suspension was placed on a carbon membrane. The carbon membrane was dried in infrared light for $20 \mathrm{~min}$ before TEM examination.

\section{Results and discussion}

The graphite nano-sheets were oxidized by a strong oxidant. Carboxyl, hydroxyl, and epoxy groups were formed on the edge of the graphene oxide. These active functional groups interact easily with other active groups. Montmorillonite has a layered structure, so a few hydroxyl groups and oxygen-containing groups exist in the edge of sheets. The functional groups in the graphene oxide interact with the groups in montmorillonite through hydrogen bonds and chemical bonds under certain conditions. The schematic of the assembly mechanism of graphene oxide and montmorillonite is shown in Fig. 1. The graphene oxide nanosheets were adsorbed on the surface of montmorillonite.

\subsection{FT-IR analysis}

The FT-IR spectra of MMT and GO/MMT were measured to confirm the structure characteristics (Fig. 2). The spectrum of MMT was characteristic of a typical smectite. Some adsorption peaks related to MMT and GO/MMT hybrids were as follows: A band at $1035 \mathrm{~cm}^{-1}$ was assigned to $\mathrm{Si}-\mathrm{O}-\mathrm{Si}$ bending vibration, and bands at 1641 and $3624 \mathrm{~cm}^{-1}$ were due to the $\mathrm{O}-\mathrm{H}$ stretching vibration of water molecules in silicate layers. The spectrum of $\mathrm{GO} /$ MMT showed a typical characteristic of hydroxyl and carbonyl groups. The characteristic peaks of MMT shifted after being combined with GO. No peak shift or only a slight shift was observed for the band at $1035 \mathrm{~cm}^{-1}$, corresponding to $\mathrm{Si}-\mathrm{O}-\mathrm{Si}$ bending vibration, in the GO/MMT composite, which indicates that the $\mathrm{Si}-\mathrm{O}-\mathrm{Si}$ bond did not interact with GO. However, the peaks (1641 and $3624 \mathrm{~cm}^{-1}$ ) of the $\mathrm{O}-\mathrm{H}$ stretching of water molecules blue-shifted from 1641 to $1636 \mathrm{~cm}^{-1}$ and from 3624 to $3621 \mathrm{~cm}^{-1}$ in the GO/MMT composite. The large shift shows that the GO sheet was adsorbed on the MMT through $\mathrm{O}-\mathrm{H}$ bond.

\subsection{Raman spectrum}

The Raman spectrum of GO, as shown in Fig. 3 displays a D-band at $1359 \mathrm{~cm}^{-1}$ and a G-band at $1594 \mathrm{~cm}^{-1}$. The D-band is associated with the structural defects related to the partially disordered structures of graphitic domains or created by the attachments of functional groups on the carbon basal plane. The G-band is attributed to the firstorder scattering of the E2g mode. The $I_{\mathrm{D}} / I_{\mathrm{G}}$ value of GO was calculated to be 0.97 , suggesting the formation of new $s p^{2}$ clusters upon reduction. The D-band and G-band reduced sharply in the GO/MMT composite (Fig. 3). What is more important, the D-band (from 1359 to $1344 \mathrm{~cm}^{-1}$ ) and G-band (from 1594 to $1574 \mathrm{~cm}^{-1}$ ) shifted significantly, suggesting a significant interaction between GO and MMT. The $I_{\mathrm{D}} / I_{\mathrm{G}}$ value of GO/MMT was calculated to be 1.13 , showing a change in the GO structure.

\subsection{XRD analysis}

The evolution of XRD patterns of MMT and GO/MMT hybrids is presented in Fig. 4. The interlayer spacing, $d$, of the pristine MMT was about $1.09 \AA$, but for the GO/MMT hybrids of different GO concentrations, the interlayer spacings were $1.25,1.20,1.30,2.25$, and $13.0 \AA$, respectively, indicating strong intercalations of GO into MMT. The interlayer space increased by $0.16,0.08$, and $0.20 \AA$, respectively, when the GO concentrations were $0.005 \mathrm{wt} \%$, $0.01 \mathrm{wt} \%$, and $0.02 \mathrm{wt} \%$; therefore, considering the hydrogen bond length of approximately $0.2 \AA$, the hydrogen bonds were formed between GO sheets and MMT sheets when the GO concentration was under $0.02 \mathrm{wt} \%$. The interlayer spacing increased significantly to $2.25 \AA$ at the addition of $0.04 \mathrm{wt} \% \mathrm{GO}$ dispersion, which is convincing evidence that $\mathrm{GO}$ has inserted into the interlayer space of MMT. When the GO concentration increased to $0.08 \mathrm{wt} \%$, the interlayer spacing shot up to $13.0 \AA$ due to the insertion of GO. This further shows the strong interaction between GO and MMT.

\subsection{XPS analysis}

Pristine MMT and hybrid GO/MMT were characterized by XPS (Table 1), and their elemental analyses show that the weight percentage of carbon (from $15.38 \mathrm{wt} \%$ to $26.59 \mathrm{wt} \%$ ) and nitrogen (from $0.78 \mathrm{wt} \%$ to $3.44 \mathrm{wt} \%$ ) increased significantly, indicating GO combined with MMT. The interaction between GO and MMT was further verified by the reduction in the contents of silicon (from $17.63 \mathrm{wt} \%$ to $11.69 \mathrm{wt} \%$ ) and aluminum (from $8.05 \mathrm{wt} \%$ 


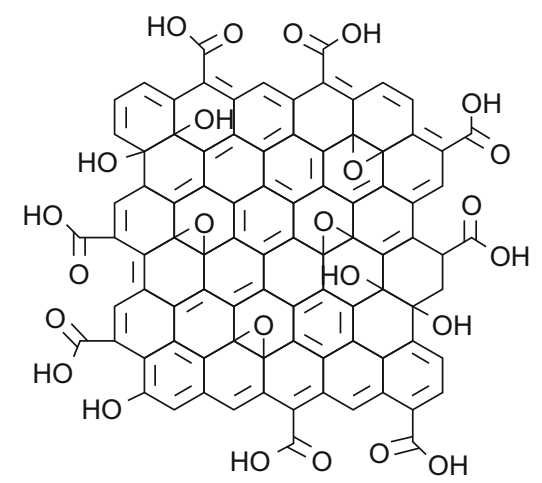

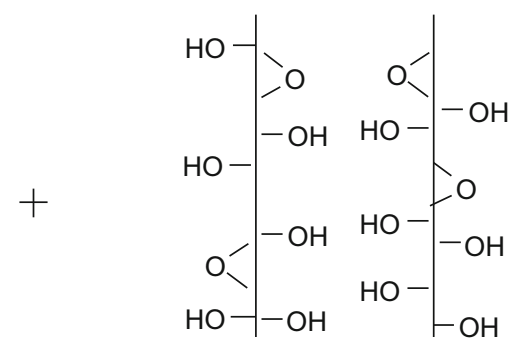

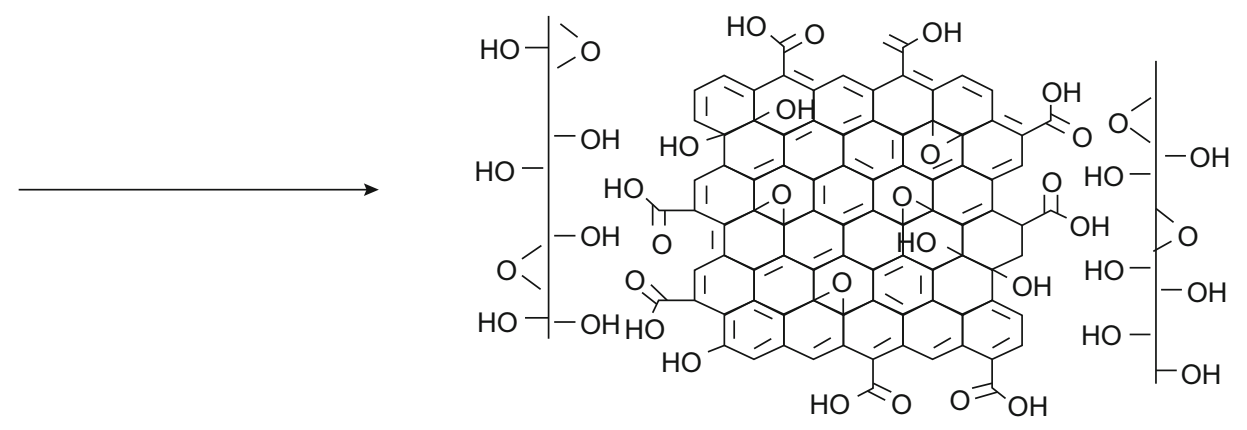

Fig. 1 Schematic of the interaction mechanism of MMT and GO

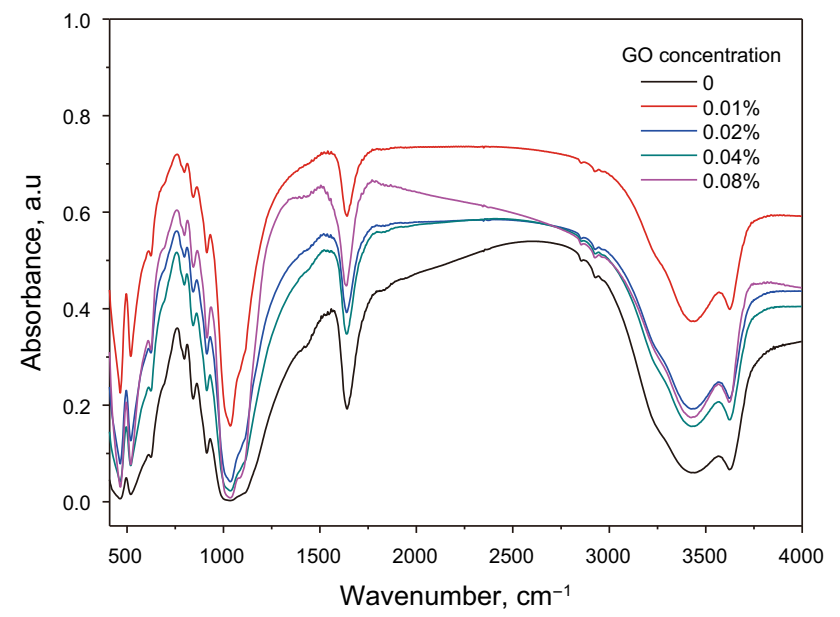

Fig. 2 FI-IR spectra of MMT and GO/MMT of different GO concentrations

to $5.48 \mathrm{wt} \%$ ) in the GO/MMT. To further illustrate this result, Fig. 5 shows the O1s XPS scans of MMT and GO/ MMT hybrid. The shift and intensity of the O1s peak changed significantly. This phenomenon convincingly demonstrates that the assembled structure was formed.

\subsection{SEM examination}

Typical SEM images of shales without and with GO are shown in Fig. 6. A large number of nano-pores and shale sheets were observed in the SEM image of fresh shale

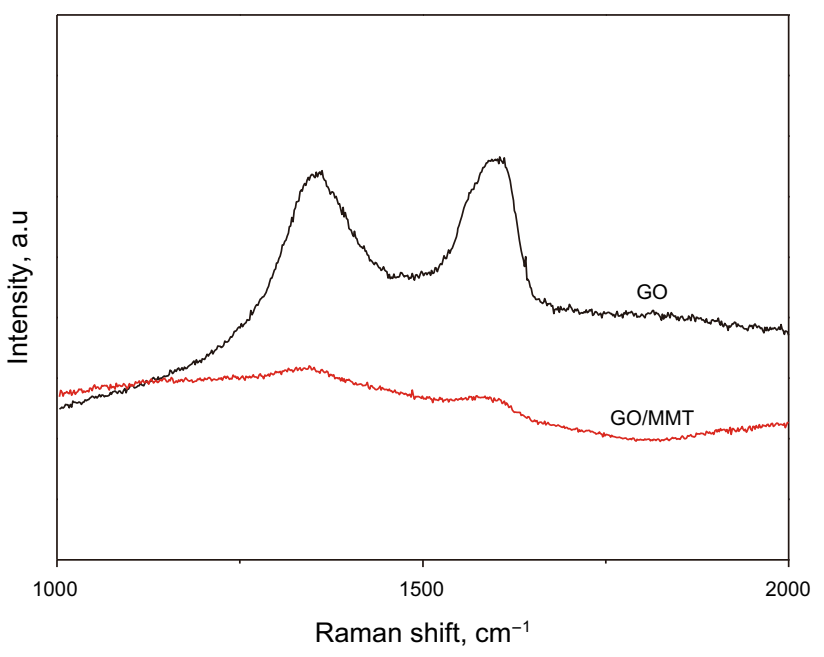

Fig. 3 Raman spectra of GO and GO/MMT hybrid

(Fig. 6a), and the edges and corners were clearly observed. After treated with the GO dispersion, the shale surface was covered with flexible sheets and no nano-pores and shale sheets were observed (Fig. 6b), indicating that the shale interacted with GO. GO sheets were flexible and thin, and obvious wrinkles were observed in the SEM image of shale treated with GO (Fig. 6b), suggesting the typical GO images were observed. These GO sheets were adsorbed on the surface of shale. The stable assembled structure was formed during the interaction process. 


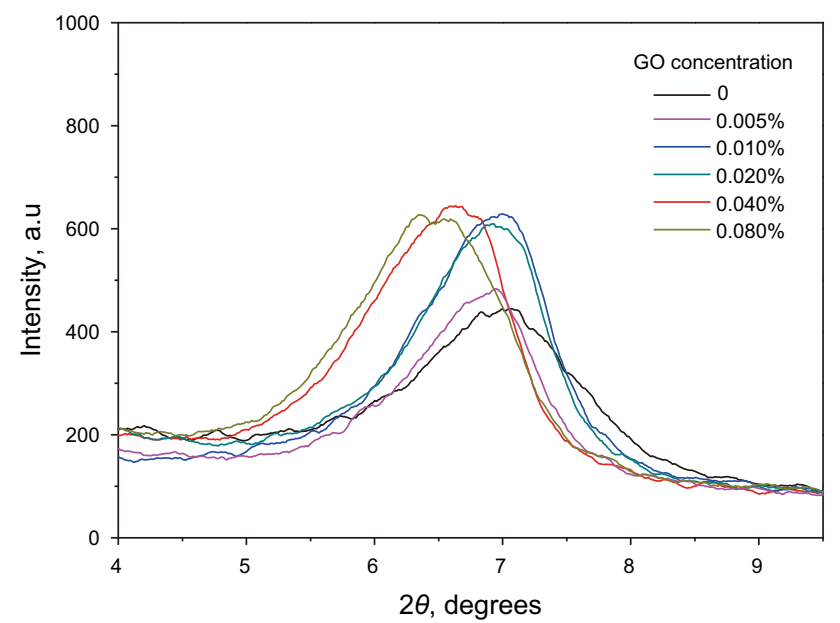

Fig. 4 XRD patterns of MMT and GO/MMT hybrids with different GO concentrations

Table 1 XPS elemental analyses of MMT and GO/MMT (GO concentration is $2.6 \mathrm{wt} \%$ )

\begin{tabular}{llllll}
\hline Sample & \multicolumn{6}{l}{ Composition, wt\% } & & & \\
\cline { 2 - 6 } & $\mathrm{C}$ & $\mathrm{O}$ & $\mathrm{N}$ & $\mathrm{Al}$ & $\mathrm{Si}$ \\
\hline MMT & 15.38 & 57.33 & 0.78 & 8.05 & 17.63 \\
GO/MMT & 26.59 & 52.84 & 3.44 & 5.48 & 11.66 \\
\hline
\end{tabular}

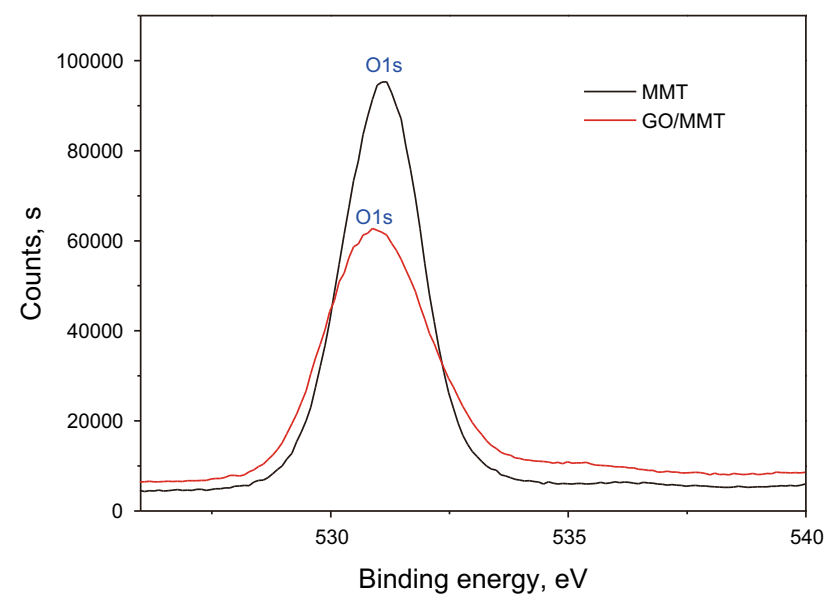

Fig. 5 XPS patterns of $\mathrm{O}$ atom in MMT and GO/MMT hybrid

\subsection{TEM examination}

A TEM image of GO/MMT is shown in Fig. 7. A small number of MMT sheets were observed in this TEM image. The size of MMT sheets were below $500 \mathrm{~nm}$, suggesting that significant hydration of MMT sheets occurred. The nano-graphene oxide sheets were tiled on the surface of MMT sheets. This observation illustrated that a stable assembly structure was formed between GO sheets and MMT sheets.

\subsection{Particle size analysis}

Figure 8 shows the particle size distributions of pristine MMT and GO/MMT suspensions. The average particle size of MMT in the pristine MMT suspension was about $500 \mathrm{~nm}$. When $0.001 \mathrm{wt} \% \mathrm{GO}$ was added into the MMT suspension. The average size of MMT particles was about $2000 \mathrm{~nm}$ due to the links between the GO sheets and MMT sheets. This is because when a small number of GO sheets (as a linker agent, $0.001 \mathrm{wt} \%$ ) linked with MMT sheets, the average size of MMT sheets enlarged. However, with the addition of GO sheets $(0.005 \mathrm{wt} \%)$, the negative charges of GO sheets repelled the negative charges of MMT sheets. The mutual repulsion force was a major factor which caused the average size of MMT sheets to reduce. An equilibrium was achieved between the repulsion force and cross-linking force by adjusting the concentration of GO sheets. Continuing to add GO sheets $(0.01 \mathrm{wt} \%)$, the crosslinking points' bulk increased; therefore, the cross-linking force played a dominant role, and the assembly between GO sheets and MMT sheets was significant. The average size of MMT sheets became larger due to the major crosslinking force. Continuing to add the GO sheets $(0.02 \mathrm{wt} \%)$, the interaction between GO sheets and MMT sheets strengthened obviously. A stable structure was formed between GO sheets and MMT sheets. This further proved that MMT sheets were successfully combined with GO sheets.

\subsection{Zeta potential analysis}

Zeta potentials of the pristine MMT suspension and $\mathrm{GO}$ / MMT suspensions are presented in Fig. 9. The negative surface charges were formed by isomorphous substitution in the octahedral layer. The addition of negative charges to the interlayer space increased the stability of the MMT suspension. The zeta potential of the pristine MMT suspension after hydration was about $-2.59 \mathrm{mV}$, presenting worse dispersion of MMT in water. The addition of GO $(0.001 \mathrm{wt} \%)$, the zeta potential of MMT reached to $-5.96 \mathrm{mV}$, and the dispersion of MMT was improved due to the cross-linking of GO sheets. Continuing to add GO solution (0.005 wt $\%)$, MMT sheets were dispersed well because of an increase in the negative charges attributed to GO sheets. The repulsion force between negative charges was the major force to keep the stability of MMT suspensions $(0.1 \mathrm{wt} \%)$. A number of hydroxyl groups and epoxy groups were at the edges of GO sheets. With the addition of GO sheets $(0.005 \mathrm{wt} \%-0.01 \mathrm{wt} \%)$ to the MMT suspension, the cross-linking points increased. Therefore, 

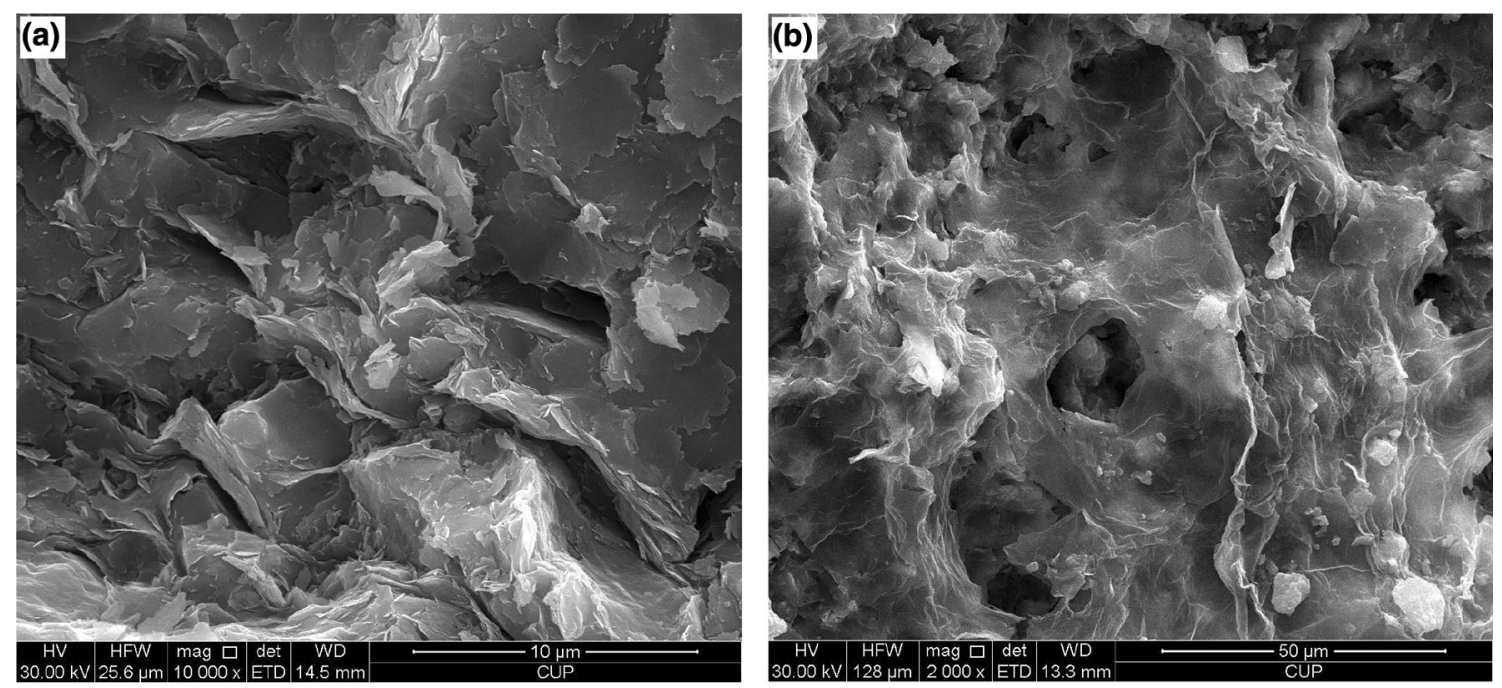

Fig. 6 SEM images of shale without and with GO. a Shale without GO. b Shale with GO

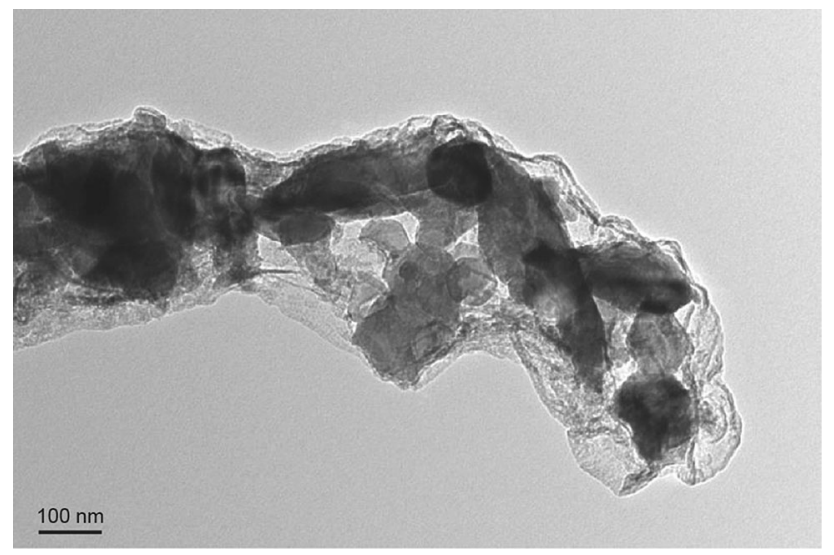

Fig. 7 TEM image of GO/MMT hybrid (GO concentration is $0.001 \mathrm{wt} \%$ and MMT concentration is $0.001 \mathrm{wt} \%$ )

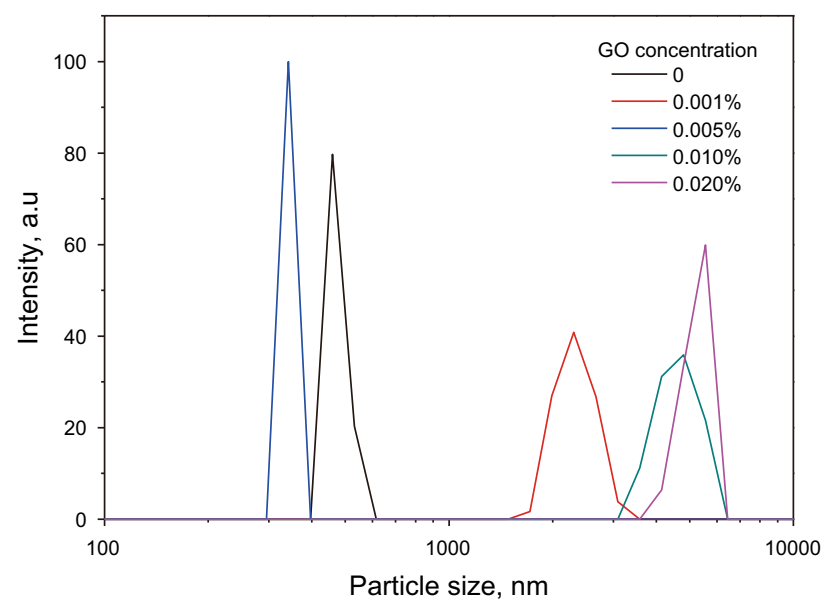

Fig. 8 Particle size distributions of pristine MMT suspension and GO/MMT suspensions of different GO concentrations (in the suspensions, the MMT concentration was $0.1 \mathrm{wt} \%$ )

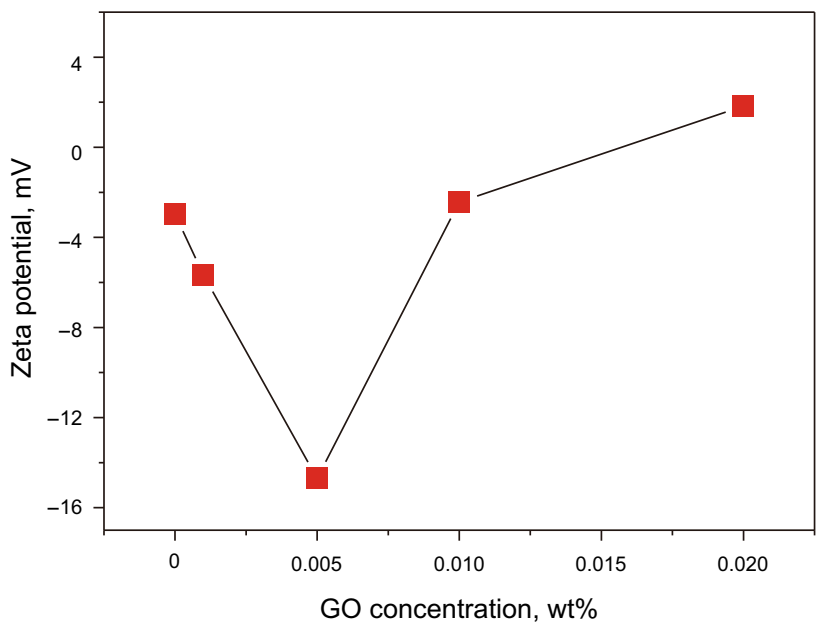

Fig. 9 Zeta potentials of MMT suspensions of different GO concentrations

strong interactions occurred between $\mathrm{GO}$ sheets (0.005 wt $\%-0.02 \mathrm{wt} \%)$ and MMT sheets $(0.1 \mathrm{wt} \%)$, indicating GO sheets were adsorbed on the surface of MMT and inserted into the interlayer space of the MMT. The assembled structure was formed between MMT sheets and GO sheets, causing a decline in the absolute value of zeta potential of MMT. This phenomenon indicated that the MMT particle began to agglomerate. These MMT aggregations assembled by GO sheets dispersed worse in water. The result of the zeta potential test was in accordance with the particle size test.

\subsection{Probable assembly mechanism}

The interaction between GO sheets and MMT sheets was studied. GO sheets and MMT sheets were repelled through 
electrostatic repulsion. There were a few negative charges at the edges of GO sheets and on the surface of MMT sheets. The hydroxyl groups of GO were attracted by the oxygen atoms through hydrogen bonds. An equilibrium was reached between repulsive and attractive forces. With enhancement of the attractive force, the equilibrium was broken, and then GO sheets were adsorbed on the surface of MMT sheets and some GO sheets inserted into the interlayer space of MMT. The adsorption and insertion of GO sheets were the main mechanism to form a stable assembly structure based on GO nano-sheets and MMT sheets.

\section{Conclusions}

Graphene oxide (GO) nano-sheets were synthesized successfully. The Raman spectrum of GO displayed a typical D-band and G-band of GO. The Raman spectrum and FTIR spectra of GO/MMT showed that interaction occurred between GO sheets and MMT sheets. XRD measurement of GO/MMT indicated that a few GO sheets had inserted into the interlayer space of MMT. XPS analysis and SEM both showed GO nano-sheets were assembled with MMT sheets. Particle size test and zeta potential test further showed that a strong interaction occurred between GO sheets and MMT sheets.

In summary, adsorption and insertion were the main interaction mechanisms between GO sheets and MMT sheets. A stable structure would be formed between GO sheets and MMT sheets because GO nano-sheets were adsorbed on the surface and inserted into the interlayer space of MMT.

Acknowledgements We would like to thank the Postdoctoral Science Foundation (H29216) and the New Method and Technology Foundation of China National Petroleum Corporation (2014A-4212) for their financial support for this work.

Open Access This article is distributed under the terms of the Creative Commons Attribution 4.0 International License (http://crea tivecommons.org/licenses/by/4.0/), which permits unrestricted use, distribution, and reproduction in any medium, provided you give appropriate credit to the original author(s) and the source, provide a link to the Creative Commons license, and indicate if changes were made.

\section{References}

Al-Bazali TM. The consequences of using concentrated salt solutions for mitigating wellbore instability in shales. J Pet Sci Eng. 2011;80:94-101. https://doi.org/10.1016/j.petrol.2011.10.005.

An Y, Jiang G, Ren Y, Zhang L, Qi Y, Ge Q. An environmental friendly and biodegradable shale inhibitor based on chitosan quaternary ammonium salt. J Pet Sci Eng. 2015;135:253-60. https://doi.org/10.1016/j.petrol.2015.09.005.
An Y, Jiang G, Qi Y, Huang X, Shi H. High-performance shale plugging agent based on chemically modified graphene. $J$ Nat Gas Sci Eng. 2016;32:347-55. https://doi.org/10.1016/j.jngse. 2016.04.048.

Bunger AP, Sarout J, Kear J, Delle Piane C, Detournay E, Josh M, et al. Experimental chemoporoelastic characterization of shale using millimeter-scale specimens. J Pet Sci Eng. 2014;118:40-51. https://doi.org/10.1016/j.petrol.2014.04.004.

Bybee K. Wellbore-stability performance of water-based-mud additives. J Pet Technol. 2009;61(9):78-9. https://doi.org/10.2118/ 0909-0078-JPT.

Díaz-Pérez A, Cortés-Monroy I, Roegiers JC. The role of water/clay interaction in the shale characterization. J Pet Sci Eng. 2007;58:83-98. https://doi.org/10.1016/j.petrol.2006.11.011.

Dreyer DR, Park S, Bielawski CW, Ruoff RS. The chemistry of graphene oxide. Chem Soc Rev. 2010;39:228-40. https://doi.org/ 10.1039/B917103G.

Gholizadeh-Doonechaly N, Tahmasbi K, Davani E. Development of high-performance water-based mud formulation based on amine derivatives. In: SPE international symposium on oilfield chemistry, 20-22 April, The Woodlands, Texas; 2009. https://doi.org/ 10.2118/121228-MS.

Geim AK. Graphene: status and prospects. Sci. 2009;324:1530-34. https://doi.org/10.1126/science.1158877.

$\mathrm{Hu}$ J, Tang S, Zhang S. Investigation of pore structure and fractal characteristics of the Lower Silurian Longmaxi shales in western Hunan and Hubei Provinces in China. J Nat Gas Sci Eng. 2016;28:522-35. https://doi.org/10.1016/j.jngse.2015.12.024.

Hummers WS, Offeman RE. Preparation of graphitic oxide. J Am Chem Soc. 1958;80:1339. https://doi.org/10.1021/ja01539a017.

Josh ML, Esteban L, Delle Piane C, Sarout J, Dewhurst DN, Clennell MB. Laboratory characterisation of shale properties. J Pet Sci Eng. 2012;88-89:107-24. https://doi.org/10.1016/j.petrol.2012. 01.023 .

Kosynkin DV, Ceriotti G, Wilson KC, Lomeda JR, Scorsone JT, Patel $\mathrm{AD}$, et al. Graphene oxide as a high-performance fluid-losscontrol additive in water-based drilling fluids. ACS Appl Mater Interfaces. 2012;4:222-7. https://doi.org/10.1021/am2012799.

Mohiuddin MA, Khan K, Abdulraheem A, Al-Majed A, Awal MR. Analysis of wellbore instability in vertical, directional, and horizontal wells using field data. J Pet Sci Eng. 2007;55:83-92. https://doi.org/10.1016/j.petrol.2006.04.021.

Novoselov KS, Geim AK, Morozov SV, Jiang D, Katsnelson MI, Grigorieva IV, et al. Two-dimensional gas of massless Dirac fermions in graphene. Nature. 2005;438:197-200. https://doi. org/10.1038/nature04233.

Park S, Ruoff RS. Chemical methods for the production of graphenes. Nat Nano. 2009;4:217-24. https://doi.org/10.1038/nnano.2009. 58.

Pearce R, Iakimov T, Andersson M, Hultman L, Spetz AL, Yakimova R. Epitaxially grown graphene based gas sensors for ultra sensitive $\mathrm{NO}_{2}$ detection. Sens Actuators B: Chem. 2011;155:451-5. https://doi.org/10.1016/j.snb.2010.12.046.

Qu Y, Lai X, Zou L, Su Y. Polyoxyalkyleneamine as shale inhibitor in water-based drilling fluids. Appl Clay Sci. 2009;44:265-8. https://doi.org/10.1016/j.clay.2009.03.003.

Rajnauth JJ. Is it time to focus on unconventional resources? In: SPETT 2012 energy conference and exhibition, 11-13 June, Port-of-Spain, Trinidad; 2012. https://doi.org/10.2118/158654MS.

Reina A, Jia X, Ho J, Nezich D, Son H, Bulovic V, et al. Large area, few-layer graphene films on arbitrary substrates by chemical vapor deposition. Nano Lett. 2009;9:30-5. https://doi.org/10. 1021/n1801827v.

Riley M, Young S, Stamatakis E, Guo Q, Ji L, De Stefano G, et al. Wellbore stability in unconventional shales: the design of a 
nano-particle fluid. In: SPE oil and gas India conference and exhibition, 28-30 March, Mumbai, India; 2012. https://doi.org/ 10.2118/153729-MS.

Robinson JT, Perkins FK, Snow ES, Wei Z, Sheehan PE. Reduced graphene oxide molecular sensors. Nano Lett. 2008;8:3137-40. https://doi.org/10.1021/n18013007.

Shadizadeh SR, Moslemizadeh A, Dezaki AS. A novel nonionic surfactant for inhibiting shale hydration. Appl Clay Sci. 2015;118:74-86. https://doi.org/10.1016/j.clay.2015.09.006.

Shivhare S, Kuru E. A study of the pore-blocking ability and formation damage characteristics of oil-based colloidal gas aphron drilling fluids. J Pet Sci Eng. 2014;122:257-65. https:// doi.org/10.1016/j.petrol.2014.07.018.

Simpson JP, Walker TO, Jiang GZ. Environmentally acceptable water-base mud can prevent shale hydration and maintain borehole stability. SPE Drill Completion. 1995;10(4):242-9. https://doi. org/10.2118/27496-PA.

Stankovich S, Dikin DA, Dommett GHB, Kohlhaas KM, Zimney EJ, Stach EA, et al. Graphene-based composite materials. Nature. 2006;442:282-6. https://doi.org/10.1038/nature04969.

Stoller MD, Park S, Zhu Y, An J, Ruoff RS. Graphene-based ultracapacitors. Nano Lett. 2008;8:3498-502. https://doi.org/10. 1021/n1802558y.

Tour JM, Bellaire TX, Schmidt HK, Cypress TX, et al. Graphene compositions and drilling fluids derived therefrom. US Patent. 2011; US 20110144386A1.

van Oort E. On the physical and chemical stability of shales. J Pet Sci Eng. 2003;38:213-35. https://doi.org/10.1016/S0920-4105(03)00 034-2.

van Oort E. A novel technique for the investigation of drilling fluid induced borehole instability in shales. In: Rock mechanics in petroleum engineering, 29-31 August, Delft, Netherlands; 1994. https://doi.org/10.2118/28064-MS

Wu Q, Xu Y, Yao Z, Liu A, Shi G. Supercapacitors based on flexible graphene/polyaniline nanofiber. Composite Films ACS Nano. 2010;4:1963-70. https://doi.org/10.1021/nn1000035.

Xiong K, Ma P, Yong F, Qian F, Yang R, Meng Y. Application of aluminum \& amino drilling fluid in drilling encountering massive coal. In: IADC/SPE Asia Pacific drilling technology conference and exhibition, 9-11 July, Tianjin, China; 2012. https://doi.org/10.2118/155897-MS
Xu Y, Bai H, Lu G, Li C, Shi G. Flexible graphene films via the filtration of water-soluble noncovalent functionalized graphene sheets. J Am Chem Soc. 2008;130:5856-7. https://doi.org/10. 1021/ja800745y.

Xu Y, Zhao L, Bai H, Hong W, Li C, Shi G. Chemically converted graphene induced molecular flattening of 5,10,15,20-tetrakis(1methyl-4-pyridinio)porphyrin and its application for optical detection of cadmium(ii) ions. J Am Chem Soc. 2009;131:13490-7. https://doi.org/10.1021/ja905032g.

Xu W, Wei C, Lv J, Liu H, Huang X, Liu T. Preparation, characterization, and properties of in situ formed graphene oxide/phenol formaldehyde nanocomposites. J Nanomater. 2013. https://doi.org/10.1155/2013/319840.

Young S, Friedheim J. Environmentally friendly drilling fluids for unconventional shale. In: Offshore Mediterranean conference and exhibition, 20-22 March, Ravenna, Italy; 2013; OMC-2013102.

Zhang C, Tiu WW, Fan W, Yang Z, Huang S, Liu T. Aqueous stabilization of graphene sheets using exfoliated montmorillonite nanoplatelets for multifunctional free-standing hybrid films via vacuum-assisted self-assembly. J Mater Chem. 2011;21(44):18011-7. https://doi.org/10.1039/C1JM13236A.

Zhang S, Qiu Z, Huang W, Cao J, Luo X. Characterization of a novel aluminum-based shale stabilizer. J Pet Sci Eng. 2013;103:36-40. https://doi.org/10.1016/j.petrol.2013.02.008.

Zhong H, Qiu Z, Huang W, Cao J. Shale inhibitive properties of polyether diamine in water-based drilling fluid. J Pet Sci Eng. 2011;78:510-5. https://doi.org/10.1016/j.petrol.2011.06.003.

Zhong H, Qiu Z, Huang W, Cao J. Poly (oxypropylene)-amidoamine modified bentonite as potential shale inhibitor in water-based drilling fluids. Appl Clay Sci. 2012;67(68):36-43. https://doi. org/10.1016/j.clay.2012.06.002.

Zhong H, Qiu Z, Sun D, Zhang D, Huang W. Inhibitive properties comparison of different polyetheramines in water-based drilling fluid. J Nat Gas Sci Eng. 2015;26:99-107. https://doi.org/10. 1016/j.jngse.2015.05.029.

Zuo L, Fan W, Zhang Y, Zhang L, Gao W, Huang Y, et al. Graphene/montmorillonite hybrid synergistically reinforced polyimide composite aerogels with enhanced flame-retardant performance. Composites Sci Technol. 2017;139:57-63. https:// doi.org/10.1016/j.compscitech.2016.12.008. 